\title{
Perfil epidemiológico e nível de conhecimento de pacientes diabéticos sobre diabetes e retinopatia diabética
}

\author{
Epidemiologic profile and level of knowledge among diabetic patients about diabetes \\ and diabetic retinopathy
}

Alana Ferreira Gomes Dias ${ }^{1}$, Márcio Fragoso Vieira², Marcussi Palata Rezende ${ }^{1}$, Akioshi Oshima ${ }^{1}$, Maria Emília Wendler Muller ${ }^{1}$, Maria Emília Xavier dos Santos ${ }^{1}$, Pedro Duraes Serracarbassa ${ }^{1}$

\section{RESUMO}

Objetivo: Conhecer o perfil epidemiológico e nível de conhecimento de pacientes diabéticos sobre diabetes e retinopatia diabética (RD).

Métodos: Estudo transversal com pacientes atendidos no Ambulatório de Retina e Vítreo do Hospital do Servidor Público Estadual de São Paulo, os quais foram divididos em dois grupos: pacientes diabéticos encaminhados para primeira avaliação oftalmológica (G1) e pacientes já acompanhados no Ambulatório (G2). Os pacientes responderam questionário e submeteram-se a exame oftalmológico. Foram utilizados os testes $x^{2}$, exato de Fisher e não paramétricos de Mann-Whitney, presumindo nível de significância de 5\%.

Resultados: A amostra total foi composta por 357 pacientes (109 no G1 e 248 no G2). A maioria dos pacientes era do sexo feminino, casada, com ensino fundamental incompleto, com média de idade de 63,3 anos e afirmou saber o que é diabetes. Entretanto, 53,2\% não sabiam qual o seu tipo de diabetes. As complicações visuais do diabetes são as mais conhecidas. Menos de um terço dos pacientes já tinha ouvido falar em RD e $77,3 \%$ não sabiam se eram acometidos. A maioria dos pacientes nunca havia recebido alguma explicação ou algum tipo de material escrito sobre diabetes ou RD. Somente $3,6 \%$ dos pacientes participavam de algum programa de educação sobre diabetes. A AV média na amostra, em logMAR, foi de 0,57 no OD e 0,51 no OE. Metade dos pacientes não tinha RD.

Conclusão: A maioria dos pacientes, apesar de receber acompanhamento multidisciplinar, apresentou pouco conhecimento sobre o diabetes e suas complicações.

Descritores: Diabetes mellitus; Retinopatia diabética; Perfil de saúde; Conhecimentos, atitudes e prática em saúde; Epidemiologia

\begin{abstract}
Purpose: Toassess the epidemiologic profile and level of knowledge of diabetic patients about diabetes and diabetic retinopathy (DR).

Methods: Cross-sectional study with patients seen at Retina and Vitreous sector of Hospital do Servidor Público Estadual de São Paulo. The subjetcs were assigned into two groups: diabetic patients sent for first ophthalmologic evaluation (G1) and patient already followed in the sector (G2). The patients answered a questionnaire and were submitted to ophthalmologic examination. It had been used chi-square $\left(x^{2}\right)$, exact of Fisher and non-parametric of MannWhitney tests, with level of significance of $5 \%$.

Results: The total sample was composed for 357 patients (109 in G1 and 248 in G2). The majority of the patients were female, married, with incomplete basic education, age average of 63.3 years and affirmed to know what it is diabetes. However, 53.2\% did not know their type of diabetes. The visual complications of diabetes are most known. Less of one third of the patients had heard of DR and $77.3 \%$ did not know if they had it. The majority of the patients had never received any explanation or lecture about diabetes or DR. Only $3.6 \%$ of the patients had participated of programs of education on diabetes. The visual acuity, in logMAR scale, was of 0.57 in OD and 0.51 in the OS. Half of the patients did not have DR.
\end{abstract}

Conclusion: The majority of the patients have low knowledge about diabetes and its complications.

Keywords: Diabetes mellitus; Diabetic retinopathy; Health profile; Health knowledge, attitudes, practice; Epidemiology

\section{INTRODUÇÃO}

0 diabetes mellitus (DM) é uma doença crônica e multissistêmica de importância crescente em saúde pública. Sua incidência e prevalência vêm aumentando, alcançando proporções epidêmicas ${ }^{(1)}$.

Acredita-se que o número de portadores de DM no mundo, estimado em aproximadamente 180 milhões em 2000, mais que dobrará até o ano de $2030^{(2)}$. No Brasil, estima-se que 7,6\%

Trabalho realizado no Hospital do Servidor Público Estadual "Francisco Morato de Oliveira" HSPE-FMO - São Paulo (SP), Brasil.

Médico, Hospital do Servidor Público Estadual "Francisco Morato de Oliveira” HSPE-FMO São Paulo (SP), Brasil.

Médico, Escola Paulista de Medicina, Universidade Federal de São Paulo - UNIFESP - São Paulo (SP), Brasil.

Endereço para correspondência: Alana Ferreira Gomes Dias. Av. Gerardo Rangel, 801- Apto. 1.301 - Sobral (CE) - CEP 62041-380 - E-mail: alana-dias@bol.com.br

Recebido para publicação em 03.12.2008

Última versão recebida em 26.07.2010

Aprovação em 18.08.2010 da população urbana entre 30 e 69 anos apresentem DM, sendo que $46 \%$ destes não sabem ser portadores ${ }^{(3)}$.

Vários órgãos podem ser acometidos pelo DM, sendo comum a presença de nefropatia, neuropatia e retinopatia ${ }^{(4)}$. As afecções oculares são frequentes e graves no paciente diabético, sendo a retinopatia diabética (RD) a mais comum, presente em 29 a 40\% dos $\operatorname{casos}^{(5)}$. É considerada a principal causa de cegueira legal em adultos em idade produtiva ${ }^{(6)}$. Estima-se que o número de pessoas com risco de sofrer perda de visão decorrente do diabetes dobre nos próximos $30 \operatorname{anos}^{(7)}$.

A RD é uma das maiores causas de cegueira irreversível no mundo, e a principal entre pessoas em idade produtiva ${ }^{(8)}$, sendo considerada uma das complicações mais temidas pelos pacientes diabéticos. Estima-se que, após 15 anos de doença, 80\% dos portadores de DM tipo 2 e 97\% dos DM tipo 1 apresentem algum grau de retinopatia ${ }^{(9-10)}$.

A eficácia do tratamento para DM com controle adequado de complicações a curto e longo prazo não depende apenas da intervenção médica. O conhecimento, assim como as crenças do paciente sobre sua condição, influenciam o processo. A 
Tabela 1. Descrição das medidas sociodemográficas para cada grupo e resultado do teste de associação

\begin{tabular}{|c|c|c|c|c|c|c|c|}
\hline \multirow[b]{3}{*}{ Variável } & \multicolumn{4}{|c|}{ Grupo } & & & \multirow[b]{3}{*}{ Valor de $\mathrm{p}$} \\
\hline & \multicolumn{2}{|c|}{ G1 } & \multicolumn{2}{|c|}{ G2 } & \multicolumn{2}{|c|}{ Total } & \\
\hline & $\mathbf{n}$ & $\%$ & $\mathbf{n}$ & $\%$ & $\mathbf{n}$ & $\%$ & \\
\hline Sexo & & & & & & & 0,819 \\
\hline Feminino & 73 & 67,0 & 163 & 65,7 & 236 & 66,1 & \\
\hline Masculino & 36 & 33,0 & 85 & 34,3 & 121 & 33,9 & \\
\hline Estado civil & & & & & & & 0,117 \\
\hline Solteiro & 4 & 3,7 & 29 & 11,7 & 33 & 9,2 & \\
\hline Casado & 73 & 67,0 & 150 & 60,5 & 223 & 62,5 & \\
\hline Viúvo & 24 & 22,0 & 53 & 21,4 & 77 & 21,6 & \\
\hline Divorciado & 8 & 7,3 & 16 & 6,5 & 24 & 6,7 & \\
\hline Escolaridade & & & & & & & 0,789 \\
\hline Fundamental incompleto & 59 & 54,1 & 134 & 54,0 & 193 & 54,1 & \\
\hline Fundamental completo & 8 & 7,3 & 14 & 5,6 & 22 & 6,2 & \\
\hline Médio incompleto & 2 & 1,8 & 7 & 2,8 & 9 & 2,5 & \\
\hline Médio completo & 17 & 15,6 & 43 & 17,3 & 60 & 16,8 & \\
\hline Superior incompleto & 1 & 0,9 & 7 & 2,8 & 8 & 2,2 & \\
\hline Superior completo & 19 & 17,4 & 40 & 16,1 & 59 & 16,5 & \\
\hline Analfabeto & 3 & 2,8 & 3 & 1,2 & 6 & 1,7 & \\
\hline Total & 109 & 100,0 & 248 & 100,0 & 357 & 100,0 & \\
\hline
\end{tabular}

Teste $x^{2}$

educação dos pacientes diabéticos e seus parentes constitui ponto fundamental na assistência médica e encoraja rastreamentos mais regulares para $\mathrm{RD}^{(11)}$.

Antes de se planejar alguma ação visando a melhoria do atendimento aos pacientes é importante conhecer seu nível de entendimento sobre o próprio estado de saúde. Portanto, este trabalho tem por objetivo estudar o perfil epidemiológico e nível de conhecimento sobre o diabetes e suas complicações, com ênfase na retinopatia diabética, presente nos pacientes diabéticos acompanhados no ambulatório de Retina e Vítreo do Hospital do Servidor Público Estadual de São Paulo (HSPE).

\section{MÉTODOS}

Realizou-se estudo do tipo transversal descritivo com pacientes atendidos no ambulatório de Retina e Vítreo do HSPE. O projeto foi aprovado pelo Comitê de Ética e Pesquisa da instituição. A amostra de conveniência foi composta por pacientes atendidos durante um período de seis meses (agosto de 2007 a janeiro de 2008).

Os pacientes incluídos no estudo foram divididos em dois grupos: o primeiro (G1) composto por pacientes com diagnóstico firmado de DM encaminhados para primeira avaliação oftalmológica e o segundo (G2), por pacientes já acompanhados no ambulatório, com ou sem diagnóstico de RD. Foram excluídos pacientes com outras retinopatias que não a causada pelo diabetes ou aqueles inaptos a responder o questionário.

Todos os pacientes foram convidados a participar voluntariamente do estudo e, após esclarecidos sobre os objetivos e a forma de realização do mesmo, os que aceitaram participar assinaram o termo de consentimento livre e esclarecido.

Todos os pacientes foram submetidos a uma entrevista por um único médico (Dias AFG), durante a qual responderam a um questionário especialmente elaborado para este estudo. O questionário contemplava idade, sexo e grau de escolaridade, prosseguindo em perguntas com respostas fechadas que buscavam avaliar o nível de conhecimento dos pacientes sobre a doença.

Ao final, todos foram submetidos à medida da acuidade visual (AV) para longe, expressa em logMAR, e tiveram suas pupilas dilatadas com colírio de tropicamida a $1 \%$ e fenilefrina

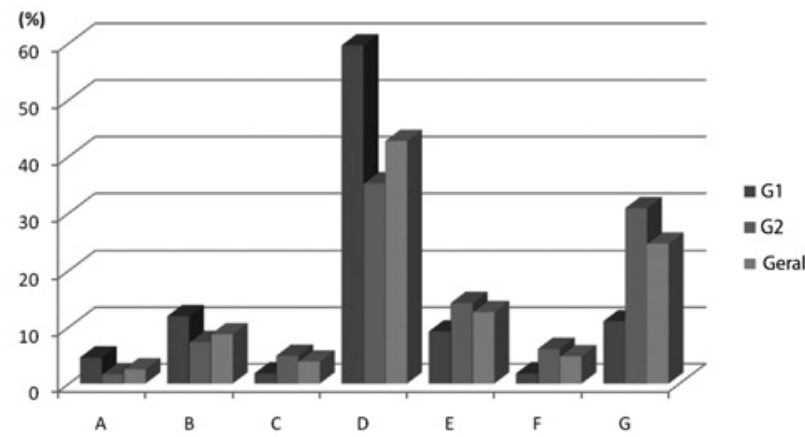

$A=$ dieta; $B=$ hipoglicemiantes orais; $C=$ insulina; $D=$ dieta + hipoglicemiantes orais; $\mathrm{E}=$ dieta + insulina; $\mathrm{F}=$ hipoglicemiantes orais + insulina; $\mathrm{G}=$ dieta + hipoglicemiantes orais + insulina

Gráfico 1. Tipos de tratamento, distribuídos por grupos.

a 10\% para realização do mapeamento de retina e biomicroscopia de fundo. Após estes exames foi realizado o diagnóstico oftalmológico do paciente.

Todas as informações foram armazenadas em banco de dados no programa Excel $2007^{\circledR}$ (Microsoft $^{\circledR}$ ) e depois analisadas no programa SPSS ${ }^{\circledR}$ versão 11.5 para Windows ${ }^{\circledR}$. Para responder os objetivos do estudo foram calculadas as frequências absolutas (contagens) e relativas (porcentuais) para as variáveis categorizadas segundo grupo e realizados testes qui-quadrado $\left(x^{2}\right)$ ou teste exato de Fisher. Para as variáveis numéricas foram calculadas as medidas resumo, expressas em média \pm desvio padrão, segundo grupo e comparadas com uso de testes não paramétricos de Mann-Whitney. Todos os testes foram realizados no nível de significância de $5 \%$.

\section{RESULTADOS}

A amostra total foi composta por 357 pacientes. O G1 foi formado por 109 pacientes e o G2 por 248. A distribuição por sexo, estado civil e grau de escolaridade dos grupos se apresenta na tabela 1. 
Tabela 2. Complicações do DM referidas pelos pacientes, distribuídas por grupo e resultado do teste de associação

\begin{tabular}{|c|c|c|c|c|c|c|c|}
\hline \multirow{3}{*}{$\begin{array}{l}\text { Quais problemas } \\
\text { o diabetes pode } \\
\text { causar? }\end{array}$} & \multicolumn{4}{|c|}{ Grupo } & \multirow{2}{*}{\multicolumn{2}{|c|}{ Total }} & \multirow[b]{3}{*}{ Valor de $\mathrm{p}$} \\
\hline & \multicolumn{2}{|c|}{ G1 } & \multicolumn{2}{|c|}{ G2 } & & & \\
\hline & $n$ & $\%$ & $n$ & $\%$ & $\bar{n}$ & $\%$ & \\
\hline Coração & & & & & & & 0,013 \\
\hline Não sabe & 44 & 40,4 & 62 & 25,0 & 106 & 29,7 & \\
\hline Não & 7 & 6,4 & 18 & 7,3 & 25 & 7,0 & \\
\hline Sim & 58 & 53,2 & 168 & 67,7 & 226 & 63,3 & \\
\hline Derrame & & & & & & & 0,001 \\
\hline Não sabe & 40 & 36,7 & 52 & 21,0 & 92 & 25,8 & \\
\hline Não & 8 & 7,3 & 9 & 3,6 & 17 & 4,8 & \\
\hline Sim & 61 & 56,0 & 187 & 75,4 & 248 & 69,5 & \\
\hline Circulação & & & & & & & 0,001 \\
\hline Não sabe & 32 & 29,4 & 33 & 13,3 & 65 & 18,2 & \\
\hline Não & 0 & 0 & 2 & 0,8 & 2 & 0,6 & \\
\hline Sim & 77 & 70,6 & 213 & 85,9 & 290 & 81,2 & \\
\hline Rins & & & & & & & $<0,001$ \\
\hline Não sabe & 29 & 26,6 & 21 & 8,5 & 50 & 14,0 & \\
\hline Não & 2 & 1,8 & 9 & 3,6 & 11 & 3,1 & \\
\hline Sim & 78 & 71,6 & 218 & 87,9 & 296 & 82,9 & \\
\hline Olhos & & & & & & & $0,207^{*}$ \\
\hline Não sabe & 4 & 3,7 & 3 & 1,2 & 7 & 2,0 & \\
\hline Sim & 105 & 96,3 & 245 & 98,8 & 350 & 98,0 & \\
\hline Total & 109 & 100,0 & 248 & 100,0 & 357 & 100,0 & \\
\hline
\end{tabular}

* = teste exato de Fisher

A média de idade geral foi de $63,3 \pm 10,3$ anos, não havendo diferença estatisticamente significativa entre os dois grupos.

Dentre os pacientes, $80,7 \%$ afirmaram saber o que é diabetes. Quando comparados por grupos, apenas 67,9\% do G1 afirmaram saber o que é diabetes contra $86,3 \%$ do G2 $(p<0,001)$. Em relação aos tipos de diabetes, somente $51 \%$ sabiam quais são os dois principais tipos. Destes, $41,3 \%$ eram do $G 1$ e $55,2 \%$ do $G 2(p=0,015)$. A maioria dos pacientes $(53,2 \%)$ não sabia qual o seu tipo de diabetes. Destes, $64,2 \%$ pertenciam ao $G 1$ e $48,4 \%$ ao $G 2(p=0,021)$. O tempo médio de diagnóstico de diabetes foi de 12,6 $\pm 9,1$ anos. No G1 foi de $8,9 \pm 9,0$ anos e no $G 2$ de $14,2 \pm 8,7$ anos $(p<0,001)$.

As formas de tratamento do diabetes utilizadas pelos pacientes no momento da entrevista são apresentadas no gráfico 1.

Foi perguntado se os pacientes sabiam qual exame é usado para verificar o nível de diabetes. Somente $61,5 \%$ do G1 afirmaram que sim. No G2 o número foi de $75,8 \%(p=0,006)$.

Setenta e sete por cento dos pacientes afirmaram ter hipertensão arterial e 44,5\%, hipercolesterolemia. Quando avaliados sobre hábitos, 6,4\% eram tabagistas, 9,2\% faziam uso de bebidas alcoólicas e apenas 40,6\% praticavam alguma atividade física. O índice de massa corpórea (IMC) médio geral foi de 28,7 \pm $5,0 \mathrm{~kg} / \mathrm{m}^{2}, 29,4 \pm 5,1$ no $\mathrm{G} 1$ e $28,4 \pm 5,0$ no $\mathrm{G} 2$ ( $\left.p>0,05\right)$.

Em relação aos médicos que prestam assistência, cada paciente é acompanhado, em média, por três especialistas. Os mais citados são oftalmologista $(96,9 \%)$, endocrinologista $(76,8 \%)$ e cardiologista $(46,2 \%)$

Perguntou-se aos pacientes se pensavam que o diabetes pode causar complicações em diversos órgãos. As respostas a esta questão estão apresentadas na tabela 2.

Em relação às complicações visuais, $75,4 \%$ pacientes acham que o diabetes pode causar baixa visão, 70,6\% no G1 e $77,4 \%$ no $G 2(p=0,171)$. Cinquenta e seis por cento acham que pode causar hemorragia. Destes, 44,0\% são do G1 e 62,1\% do $G 2(p=0,002)$. Quarenta e dois por cento dos pacientes acham que pode causar catarata. Destes, $39,4 \%$ estavam no G1 e $44,0 \%$ no $G 2(p=0,428)$. Já em relação ao glaucoma, $46,2 \%$ acham que pode ser causado pelo diabetes, 40,4\% no G1 e

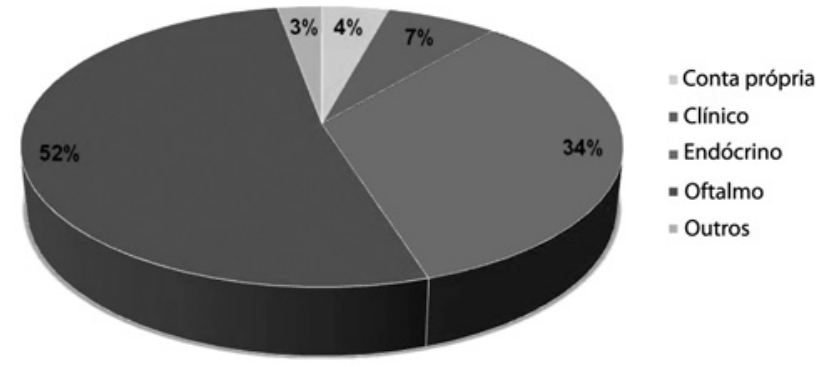

Gráfico 2. Origem do encaminhamento para realização do exame de fundo de olho, segundo informações dos próprios pacientes.

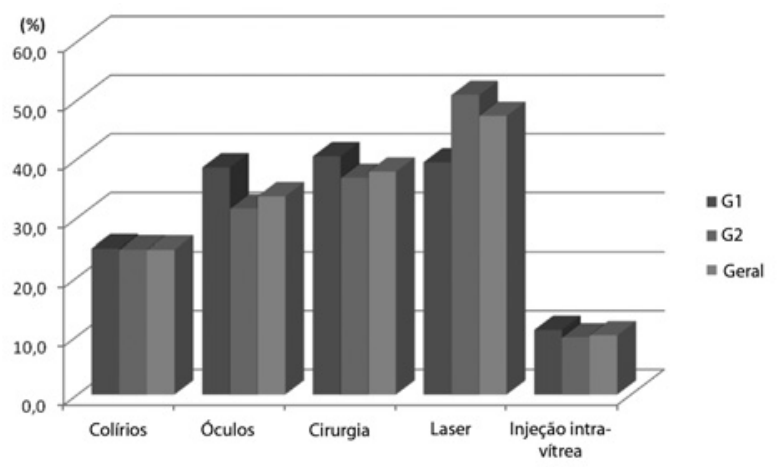

Gráfico 3. Tipos de tratamento referidos pelos pacientes, segundo os grupos.

$48,8 \%$ no G2 ( $p=0142$ ). Sobre a cegueira, $94,7 \%$ acham que é uma complicação do DM. Destes, $89,9 \%$ são do G1 e 96,8\% do $\mathrm{G} 2(\mathrm{p}=0,008)$. Somente $1 \%$ acha que o diabetes pode causar descolamento de retina.

Foi perguntado aos pacientes quais especialistas os haviam encaminhado para realização do exame de fundo de olho. As respostas a esta pergunta são apresentadas no gráfico 2 . 


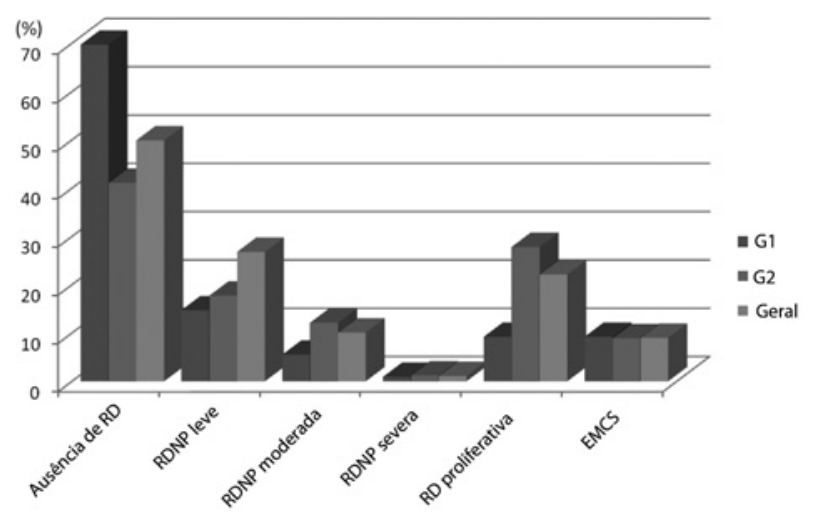

Gráfico 4. Diagnóstico oftalmológico final dos pacientes, distribuídos por grupos.

Perguntados se haviam ouvido falar sobre retinopatia diabética, somente $32,5 \%$ dos pacientes afirmaram que sim. Destes, $22 \%$ eram do $G 1$ e $37,1 \%$ do $G 2(p=0,005)$. Em relação ao conhecimento de serem portadores de retinopatia diabética, $77,3 \%$ referiram não saber; $89,0 \%$ do G1 e 72,2\% do G2.

Em relação aos tipos de tratamento para complicações visuais do diabetes, $42 \%$ não conheciam nenhum tipo de tratamento, sendo $46(42,2 \%)$ do G1 e $104(41,9 \%)$ do G2. Os outros tipos de tratamento são mostrados no gráfico 3.

Foi perguntado aos pacientes se já haviam recebido alguma explicação ou assistido palestra sobre DM de algum profissional de saúde. No total, 59,1\% dos pacientes responderam que não, 39,2\% que sim e 1,7\% não sabiam. Quando comparados os grupos, os resultados foram de 67,0\%, 32,1\% e 0,9\% no G1; e $55,6 \%, 42,3 \%$ e $2,0 \%$ no $G 2$ ( $p=0,122)$.

Quando perguntado se já haviam recebido algum tipo de material escrito sobre DM, 66,1\% disseram que não, 33,6\% que sim e 0,3\% não sabiam. Analisando-se por grupos, $72,5 \%$ disseram que não e $27,5 \%$ que sim no G1; e 63,3\% afirmaram que não, $36,3 \%$ que sim e $0,4 \%$ que não sabiam no $G 2(p=0,209)$.

Após indagados se já haviam procurado por conta própria se informar sobre DM, 43,5\% dos pacientes afirmaram que sim. Destes, 33,3\% eram do G1 e 48,0\% do G2 ( $p=0,010)$.

Foi perguntado aos pacientes se haviam participado ou participavam de algum programa de educação sobre DM. Setenta e cinco por cento dos pacientes afirmaram que não, sendo $79,8 \%$ do G1 e 73,8\% do G2. Três por cento dos pacientes responderam que participavam, sendo $2,8 \%$ do G1 e 3,6\% do G2. Vinte e um por cento disseram que já haviam participado. Destes, $17,4 \%$ eram do $G 1$ e $22,6 \%$ do $G 2$ ( $p=0,474)$.

A acuidade visual média na amostra, em logMAR, foi de $0,57 \pm 0,82$ no OD e 0,51 $\pm 0,71$ no OE. No G1 foi de 0,33 $\pm 0,52$ no OD e 0,33 $\pm 0,53$ no OE. No G2 foi de 0,67 $\pm 0,91$ no OD e $0,58 \pm 0,76$ no OE $(p<0,001)$

O diagnóstico final dos pacientes está exposto no gráfico 4.

\section{DISCUSSÃO}

A amostra foi constituída, em sua maioria, por pessoas do sexo feminino, casadas e com ensino fundamental incompleto. Não houve diferença estatisticamente significante entre os grupos em relação a estas características.

A maioria dos pacientes respondeu que sabia o que era diabetes. Apesar dessa afirmativa, cerca da metade não sabia quantos tipos de diabetes existem, nem tampouco o seu próprio tipo de diabetes. Uma grande parcela dos pacientes não sabia qual exame é utilizado para verificar o controle do diabetes. Nessas questões, houve significativa superioridade do G2 em relação ao G1. Estes dados revelam uma importante lacuna no conhecimento sobre informações básicas a respeito da doença.

O tratamento clínico da RD é baseado numa dieta saudável, na realização de exercícios físicos regulares, na eliminação dos fatores de risco (como o tabagismo), no controle adequado da glicemia e da hipertensão arterial(12). A despeito disso, a maior parte dos pacientes tem hipertensão, cerca da metade tem hipercolesterolemia e menos da metade pratica alguma atividade física.

Dentre as complicações do diabetes, as visuais são as mais relatadas ${ }^{(13)}$. Delas, a cegueira e a baixa da acuidade visual são as mais conhecidas. Vale ressaltar que somente metade dos pacientes do G1 sabia que o diabetes pode causar doenças cardíacas ou acidente vascular cerebral.

Um estudo nacional publicado em 2005(10), atentou para as percepções de pacientes atendidos em associação de diabetes em Rio Claro (SP) a respeito do tratamento da RD. Concluiu-se que existe um acentuado desconhecimento sobre RD, tratamento a laser e gravidade da afecção. Dentre os nossos pacientes, somente um terço já tinha ouvido falar em RD e 77,3\% não sabiam se eram acometidos ou não.

Assim como em todas as doenças crônicas, fornecer aos pacientes informação e educação é a melhor maneira de controlar o DM e prevenir suas complicações. Pensando nisso, realizou-se um estudo para investigar o conhecimento sobre DM e seus fatores associados em uma população de diabéticos da Turquia. Apenas $28,7 \%$ dos pacientes tinham sido informados sobre o DM( ${ }^{(14)}$. Na nossa população, a porcentagem correspondente foi de $39 \%$.

Um estudo realizado com um grupo de pacientes diabéticos em um hospital de Munique (Alemanha) procurou saber o quanto esses pacientes sabiam sobre sua doença. Concluiu-se que esforços para assegurar educação sobre diabetes aos pacientes acometidos por esta enfermidade é essencial para reduzir os riscos de complicações ${ }^{(15)}$.

Os pacientes da nossa amostra eram acompanhados em média por três médicos, o que, teoricamente, aumentaria a transmissão de informações sobre a doença. Entretanto, em estudo recente, demonstrou-se que o conhecimento médico das medidas preventivas e de periodicidade do exame para diagnóstico de RD apresenta-se distante do ideal(16).

Pesquisadores procuraram avaliar o grau de conhecimento sobre RD entre pacientes diabéticos acompanhados em um hospital de Florianópolis (SC). Observou-se que parte significativa dos pacientes não recebiam informações sobre RD de médicos ${ }^{(17)}$.

Um estudo realizado em Israe|(18) avaliou a relação entre RD, controle glicêmico, fatores de risco e educação dos pacientes. Afirmou-se que o oftalmologista tem papel importante na educação dos pacientes, reforçando a necessidade de controles glicêmicos, lipídicos e pressóricos adequados como parte do tratamento da RD.

Para avaliar como pacientes diabéticos percebem a retinopatia, o rastreamento para lesões patológicas e o seu próprio papel na prevenção da cegueira, realizou-se um estudo o qual demonstrou que um grupo de pacientes inscrito em um programa educacional sobre diabetes obteve melhores resultados em todas as perguntas a respeito de RD e tinha mais consciência da importância da prevenção(19).

Apesar de já existirem evidências de que a educação de pacientes diabéticos e seus parentes constitui um ponto fundamental tanto para encorajar rastreamentos mais regulares para RD quanto na prevenção e tratamento da $\mathrm{RD}^{(14,19-23)}$, a maioria dos pacientes na nossa amostra relata que nunca havia recebido alguma informação ou palestra sobre diabetes de algum profissional de saúde, bem como não haviam recebido algum tipo de material informativo sobre DM. Uma grande 
parte da nossa amostra também nunca havia participado de programas de educação sobre DM.

\section{CONCLUSÃO}

Apesar de terem acompanhamento multidisciplinar, os pacientes da amostra apresentam baixo nível de conhecimento sobre o DM e suas complicações. Dentre estes, os pacientes já acompanhados apresentaram melhor nível de conhecimento, embora ainda distante do ideal.

\section{REFERÊNCIAS}

1. Sociedade Brasileira de Diabetes. Consenso Brasileiro sobre Diabetes 2002: diagnóstico e classificação do diabetes melito e tratamento do diabetes melito do tipo 2. Rio de Janeiro: Diagraphic; 2003

2. World Health Organization. Diabetes: diabeties facts. Fact sheet № 312. 2008. November 2009. Disponivel em: http:// www.who.int/mediacentre/factsheets/fs312/en/ index.html

3. Malerbi DA, Franco L. Multicenter study of the prevalence of diabetes mellitus and impaired glucose tolerance in the urban Brazilian population aged 30-69 yr. The Brazilian Cooperative Group on the Study of Diabetes Prevalence. Diabetes Care. 1992:15(11):1509-16

4. Nehemy MB. Retinopatia diabética. Arq Bras Oftalmol. 1998;61 (3):366-70.

5. Klein BE, Klein R, Wang Q, Moss SE. Older-onset diabetes and lens opacities. The Beaver Dam Eye Study. Ophthalmic Epidemiol. 1995:2(1):49-55.

6. Barber AJ. A new view of diabetic retinopathy: a neurodegenerative disease of the eye. Prog Neuropsychopharmacol Biol Psychiatry. 2003;27(2):283-90. Review.

7. Wild S, Roglic G, Green A, Sicree R, King H. Global prevalence of diabetes: estimates for the year 2000 and projections for 2030. Diabetes Care. 2004:27(5):1047-53.

8. Moreira Júnior CA, Ávila M. Retinopatia diabética. In: Moreira Júnior CA, Ávila M. Retina e vítreo. Rio de Janeiro: Cultura Médica: 2000 p.61-76.

9. Klein R, Klein BE, Moss SE, Davis MD, DeMets DL. The Wisconsin epidemiologic study of diabetic retinopathy. II. Prevalence and risk of diabetic retinopathy when age at diagnosis is less than 30 years. Arch Ophthalmol. 1984;102(4):520-6.

10. Silva VB, Temporini ER, Moreira Filho DC, Kara-Jose N Tratamento da retinopatia diabética: percepções de pacientes em Rio Claro (SP) - Brasil. Arq Bras Oftalmol. 2005;68(3):363-8.
11. Treatment techniques and clinical guidelines for photocoagulation of diabetic macular edema. Early Treatment Diabetic Retinopathy Study Report Number 2. Early Treatment Diabetic Retinopathy Study Research Group. Ophthalmology. 1987:94(7):761-74

12. Abujamra S. Retinopatia diabética não-proliferativa. In: Lavinsky J, organizador. Doenças prevalentes da retina e vítreo. Rio de Janeiro: Cultura Médica; 2002. p.471-87.

13. Aldington SJ, Kohner EM, Meuer S, Klein R, Sjolie AK. Methodology for retina photography and assessment of diabetic retinopathy: the EURODIAB IDDM complications study. Diabetologia. 1995;38(4):437-44.

14. Caliskan D, Ozdemir O, Ocaktan E, Idil A. Evaluation of awareness of diabetes mellitus and associated factors in four health center areas. Patient Educ Couns. 2006;62(1):142-7

15. Blankenfeld H, Mielck A, Schumm-Draeger PM, Siegmund T. [How much do inpatient treated diabetics know about their disease?]. Gesundheitswesen. 2006; 68(8-9):557-65. German

16. Preti RC, Saraiva F, Junior JA, Takahashi WY, da Silva ME. How much information do medical practitioners and endocrinologists have about diabetic retinopathy? Clinics (Sao Paulo). 2007;62(3):273-8.

17. Adam Netto A Trindade SP Nível de conhecimento de pacientes diabéticos sobre a retinopatia diabética. Rev Bras Oftalmol. 2004;63(2):130-9

18. Axer-Siegel R, Herscovici Z, Gabbay M, Mimouni K, Weinberger D, Gabbay U. The relationship between diabetic retinopathy, glycemic control, risk factor indicators and patient education. Isr Med Assoc J. 2006;8(8):523-6. Comment in: Isr Med Assoc J. 2006;8(8):568-9.

19. Austin MM. Diabetes educators: partners in diabetes care and management. Endocr Pract. 2006;12 Suppl 1:138-41

20. Basch CE, Walker EA, Howard CI, Shamoon H, Zybert P. The effect of health education on the rate of ophthalmic examinations among African Americans with dia0betes mellitus. Am J Public Health. 1999;89(12):1878-82.

21. Golay A, Lagger G, Chambouleyron M, Carrard I, Lasserre-Moutet A. Therapeutic education of diabetic patients. Diabetes Metab Res Rev. 2008;24(3): 192-6. Review.

22. McCarty CA, Lloyd-Smith CW, Lee SE, Livingston PM, Stanislavsky YL, Taylor HR. Use of eye care services by people with diabetes: the Melbourne Visual Impairment Project. Br J Ophthalmol. 1998;82(4):410-4. Erratum in: Br J Ophthalmol 1998;82(5):591.

23. Trento M, Bajardi M, Borgo E, Passera P, Maurino M, Gibbins R, et al. Perception of diabetic retinopathy and screening procedures among diabetic people. Diabet Med. 2002:19(10):810-3.

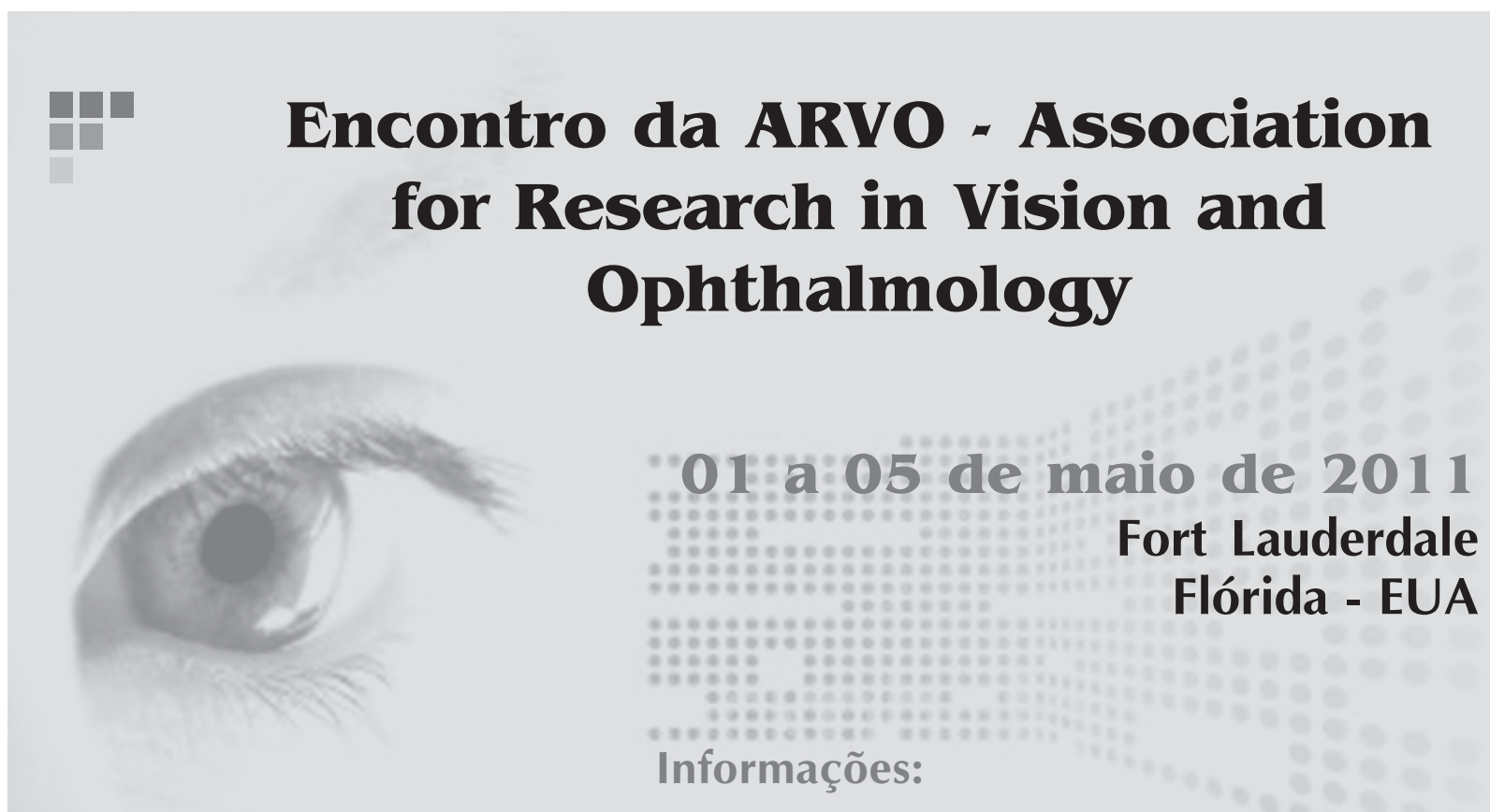

Site http://www.arvo.org/eweb/startpage.aspx?site=arvo2 